\title{
Consumo de fibras e dificuldades alimentares na infância: alimentos contribuintes e fatores associados
}

\section{Fiber consumption and feeding difficulties in childhood: food sources and associated factors}

\author{
Luana Romão Nogueira, Paula Louro Silva, Juliana Masami Morimoto, \\ Raquel Ricci, Priscila Maximino, Mauro Fisberg
}

Como citar este artigo: NOCUEIRA, LUANA R; SILVA, PAULA L.; MORIMOTO, JULIANA M.; RICCl, RAQUEL; MAXIMINO, PRISCILA: FISBERG, MAURO

Consumo de fibras e dificuldades alimentares na infância: alimentos contribuintes e fatores associados. Revista Saúde (Sta. Maria). 2020; 46 (2).

\section{Autor correspondente:}

Nome: Luana Romão Nogueira

E-mail: lvanarnog@gmail.com

Telefone: (1 1 ) 2155-9358

Formação Profissional:

Nutricionista, mestrado em nutrição em saúde pública pela

Faculdade de Saúde Pública -

USP, São Paulo, SP, Brasil.

Filiação Institucional: Instituto PENSI

Endereço para correspondência: Av. Angélica

Bairro: Consolação

Cidade: São Paulo

Estado: São Paulo

CEP: 0 1228-200

Data de Submissão:

22/07/2020

Data de aceite:

26/08/2020

Conflito de Interesse: Não há conflito de interesse

\section{(cc) $\mathrm{BY}-\mathrm{NC}-\mathrm{ND}$}

\section{RESUMO}

Objetivos: Descrever o consumo de fibras por crianças com dificuldades alimentares (DA) de acordo com a recomendação da Sociedade Brasileira de Pediatria (SBP), identificar e descrever os cinco alimentos que mais contribuem para o consumo de fibras, bem como verificar os fatores associados ao consumo de fibras dentro da recomendação. Métodos: Trata-se de um estudo observacional retrospectivo, baseado em utilização de dados de prontuários e com amostragem montada por conveniência. Os cinco alimentos que mais contribuíram para o consumo de fibras (totais, solúveis e insolúveis) foram apresentados de acordo com o percentual de contribuição. Os fatores associados ao consumo de fibras dentro da recomendação foram verificados utilizando regressão logística. Resultados: A maioria das crianças $(53,8 \%)$ apresentou consumo abaixo da recomendação da SBP. Os maiores contribuintes para o consumo de fibras totais foram pães, arroz e cereais, seguido de frutas, feijões e outras leguminosas, hortaliças e sucos de frutas. Os contribuintes de fibras solúveis e insolúveis são semelhantes ao rank de fibras totais. Escolaridade dos pais apresentou associação positiva com consumo de fibras dentro da recomendação (OR: 5,30; IC $95 \% 1,08-25,99 ; p=0,039)$, enquanto uso de distração durante a refeição e falta de autonomia da criança para se servir apresentaram associação negativa (OR: 0,43; IC 95\% 0,19-0,96; p=0,040 e OR: 0,44; IC 95\% 0,20-0,98; $p=0,045$, respectivamente). Conclusão: A maioria das crianças com DA apresentou consumo de fibras abaixo da recomendação. A maior parte dos alimentos contribuintes apresenta boa qualidade nutricional. Os fatores associados ao consumo de fibras foram escolaridade dos pais, uso de distração durante a refeição e autonomia da criança para se servir. Dessa forma, a dinâmica das refeições e o papel dos pais na alimentação são importantes e devem ser avaliados e orientados durante 0 acompanhamento das DA.

PALAVRAS-CHAVE: Fibra alimentar; Alimentação infantil; Seletividade alimentar; Consumo alimentar.

\section{ABSTRACT}

Objectives: Todescribe fiber intake by children with feeding difficulties(FD)according to the recommendation of the Brazilian Society of Pediatrics (SBP), identify and describe the five foods that most contribute to fiber intake, and verify the associated factors with fiber consumption within the recommendation. Methods: This is a retrospective observational study, based on the use of data from medical records and with sampling assembled for convenience. The five foods that contributed most to fiber consumption (total, soluble and insoluble) were presented according to the percentage of contribution. The factors associated with fiber consumption within the recommendation were verified using logistic regression. Results: Most children (53.8\%) had a consumption below the SBP recommendation. The major contributors to the total fiber intake were breads, rice and cereals, followed by fruits, beans and other pulses, vegetables and fruit juices. The contributors of soluble and insoluble fibers are similar to the total fiber rank. Parents' education showed a positive association with fiber consumption within the recommendation (OR: 5.30; $95 \% \mathrm{Cl} 1.08-25.99 ; p=0.039$ ), while using distraction during the meal and lack of child autonomy to serve themselves showed a negative association (OR: $0.43 ; 95 \% \mathrm{Cl} 0.19-0.96 ; p=0.040$ and OR: $0.44 ; 95 \%$ $\mathrm{Cl} 0.20-0.98 ; p=0.045$, respectively). Conclusion: Most children with FD presented fiber consumption below the recommendation. Most of food sources have good nutritional quality. The factors associated with fiber consumption were parents' education, use of distraction during the meal and the child autonomy to serve themselves. Thus, the meals dynamics and the parentes role in food are important and should be evaluated and guided during the FD monitoring. 


\section{INTRODUÇÃO}

As fibras dietéticas referem-se aos carboidratos não-digeríveis, podendo ser classificadas como solúveis e insolúveis. As solúveis retardam o esvaziamento gástrico e o trânsito intestinal, bem como reduzem o colesterol. Já as fibras insolúveis possuem a função de acelerar o trânsito intestinal, assim como aumentar o bolo fecal' ${ }^{1}$. As fibras são importantes componentes de uma alimentação saudável, visto que o consumo adequado, a longo prazo, pode reduzir o risco do desenvolvimento de doenças crônicas, como hipertensão arterial e diabetes melito ${ }^{2,3}$. Além disso, é fator de proteção para alguns problemas gastrointestinais, como a constipação intestinal, que pode afetar o bem-estar e até a performance escolar de crianças ${ }^{4}$.

Os principais alimentos fontes de fibras são frutas, hortaliças e cereais integrais, no entanto, é comum que estes alimentos sejam pouco aceitos por crianças, especialmente em casos de seletividade alimentar ${ }^{5}$. Mais da metade dos pais descrevem que seus filhos são seletivos ou comem pouco e estima-se que de 8 a $50 \%$ das crianças possuam dificuldades alimentares $(\mathrm{DA})^{6}$. Define-se DA como todos os episódios em que a criança possui uma menor aceitação alimentar e que interfere negativamente todo o processo de sua alimentação ${ }^{6}$. Essa situação pode ser apenas devido às adaptações da alimentação infantil ou pode ser apresentada até a vida adulta, sendo desenvolvida em qualquer fase da vida da criança ${ }^{7}$.

Assim, considerando os aspectos expostos, os objetivos do presente estudo são: (i) descrever o consumo de fibras por crianças com dificuldades alimentares de acordo com a recomendação da Sociedade Brasileira de Pediatria (SBP); (ii) identificar e descrever os cinco alimentos que mais contribuem para o consumo de fibras nessa população; (iii) verificar os fatores associados ao consumo de fibras dentro da recomendação.

\section{MÉTODO}

Trata-se de um estudo observacional retrospectivo, baseado em utilização de dados de prontuários já coletados e autorizados para uso científico em projeto de pesquisa anterior (registrado sob CAAE 32939314.0.0000.5567). O projeto foi realizado a partir da análise de 145 prontuários de pacientes diagnosticados com DA (excluindo casos de transtornos alimentares psiquiátricos de acordo com o diagnóstico do DSM $-5^{8}$ ) de ambos os sexos, com idade de 0 a 18 anos atendidos no Centro de Dificuldades Alimentares (CDA). O diagnóstico do paciente é baseado na classificação de Kerzner ${ }^{9}$, com adaptações. O centro de referência considera a classificação das DA em seletividade alimentar, apetite limitado e fobia alimentar. Maiores detalhes sobre o protocolo de atendimento do serviço podem ser verificados em publicação anterior ${ }^{10}$.

O centro é parte do Instituto de PENSI - Pesquisa e Ensino em Saúde Infantil/Hospital Infantil Sabará/Fundação José Luiz Egydio Setúbal, localizado em São Paulo, Brasil. A amostragem foi montada por conveniência, com a inclusão 
de todos os pacientes atendidos entre abril de 2016 a dezembro de 2018. Todos os pacientes apresentaram um termo de consentimento por escrito assinado pelo responsável e o projeto foi aprovado pelo Comitê de Ética em Pesquisa (CAAE 12648119.9.0000.5567).

Os dados foram obtidos com base na entrevista realizada com a mãe e/ou com o pai durante o primeiro atendimento multiprofissional da criança, após análise de prontuários. Os dados de alimentação foram obtidos por meio do inventário de alimentos (IA), ferramenta desenvolvida pela equipe do serviço no qual a nutricionista deve realizar a história alimentar, solicitar o registro alimentar de 3 dias, além de coletar o volume de líquidos do paciente durante a primeira consulta ${ }^{11}$.

Posteriormente, os dados alimentares do primeiro registro alimentar foram digitados no Nutrition Data System for Research (NDS-R) (versão 2007, Nutrition Coordinating Center, Universidade de Minnesota, Minneapolis, EUA) que consiste em um programa de análise dietética que conta com mais de 165 nutrientes e outros componentes, 18000 tipos de alimentos, além de permitir a exportação dos dados para análise em diferentes dimensões (nutrientes, alimentos, refeições, ingredientes).

A tabulação das outras informações foi realizada com o auxílio do programa Microsoft Excel 2013. O consumo de fibras em gramas foi classificado de acordo com a recomendação SBP ${ }^{1}$ (idade da criança em anos + $5=$ recomendação de gramas de fibras por dia; máximo de 25 gramas por dia). Para a análise estatística descritiva, as variáveis qualitativas foram apresentadas por meio de frequências relativas e absolutas e variáveis quantitativas foram apresentadas por meio de médias e desvio padrão. Para as diferenças entre os grupos que ficaram abaixo ou dentro da recomendação de fibras e as variáveis de interesse utilizou-se o qui-quadrado de Pearson. Os cinco alimentos que mais contribuíram para o consumo de fibras totais, fibras solúveis e fibras insolúveis foram apresentados por ordem crescente de acordo com 0 \% de contribuição. Os fatores associados ao consumo dentro da recomendação de fibras foram verificados utilizando regressão logística por meio do software Stata (versão 13.0, StataCorp, College Station, TX, EUA). Os resultados foram apresentados na forma de odds ratio (OR) e intervalos e confiança de 95\% (IC 95\%). Para todas as análises considerouse o nível de significância de $5 \%$.

\section{RESULTADOS}

Foram incluídos dados de 145 crianças com DA com idade média de 4,7士2,9 anos. A maioria era do sexo masculino $(65,5 \%)$ e não atingiu a recomendação de consumo de fibras (53,8\%). A tabela 1 apresenta a descrição de variáveis demográficas, escolaridade dos pais, aleitamento materno exclusivo (AME), dinâmica de refeições e número de alimentos aceitos de acordo com o consumo de fibras abaixo ou dentro da recomendação. Houve diferenças 
estatisticamente significativas para escolaridade dos pais $(p=0,024)$, uso de distração durante a refeição $(p=0,037)$ e autonomia da criança para se servir $(p=0,042)$.

Tabela 1 - Distribuição das variáveis de acordo com a recomendação de consumo de fibras. São Paulo, 2019.

\begin{tabular}{|c|c|c|c|c|}
\hline & $\mathrm{n}(\%)$ & $\begin{array}{l}\text { Consumo de } \\
\text { fibras abaixo da } \\
\text { recomendação } \\
\mathrm{n}(\%)\end{array}$ & $\begin{array}{l}\text { Consumo de } \\
\text { fibras dentro da } \\
\text { recomendação } \\
\mathrm{n}(\%)\end{array}$ & Valor de $p$ \\
\hline \multicolumn{5}{|l|}{ Sexo $(n=145)$} \\
\hline Feminino & $50(34,5)$ & $32(64,0)$ & $18(36,0)$ & 0,074 \\
\hline Masculino & $95(65,5)$ & $46(48,4)$ & $49(51,6)$ & \\
\hline \multicolumn{5}{|l|}{ Escolaridade dos pais ( $n=138)$} \\
\hline Ensino médio & $10(7,3)$ & $2(20,0)$ & $8(80,0)$ & 0,024 \\
\hline Ensino superior & $128(92,7)$ & $73(57,0)$ & $55(43,0)$ & \\
\hline \multicolumn{5}{|c|}{ Aleitamento materno exclusivo $(n=138)$} \\
\hline Não realizou & $34(24,6)$ & $19(55,9)$ & $15(44,1)$ & 0,830 \\
\hline$<6$ meses & $60(43,5)$ & $30(50,0)$ & $30(50,0)$ & \\
\hline$\geq 6$ meses & $44(31,9)$ & $24(54,5)$ & $20(45,5)$ & \\
\hline \multirow{2}{*}{\multicolumn{5}{|c|}{$\begin{array}{l}\text { Diagnóstico de dificuldade } \\
\text { alimentar }(n=145)\end{array}$}} \\
\hline & & & & \\
\hline Apetite limitado & $34(23,4)$ & $20(58,8)$ & $14(41,2)$ & 0,676 \\
\hline Fobia alimentar & $5(3,4)$ & $2(40,0)$ & $3(60,0)$ & \\
\hline Seletividade alimentar & $97(66,9)$ & $50(51,5)$ & $47(48,5)$ & \\
\hline Outros & $9(6,3)$ & $6(66,7)$ & $3(33,3)$ & \\
\hline \multicolumn{5}{|c|}{ Doença orgânica associada ( $n=145)$} \\
\hline Sim & $60(41,4)$ & $33(55,0)$ & $27(45,0)$ & 0,807 \\
\hline Não & $85(58,6)$ & $45(52,9)$ & $40(47,1)$ & \\
\hline \multicolumn{5}{|l|}{ Constipação (n=100) } \\
\hline Sim & $32(32,0)$ & $18(56,3)$ & $14(43,7)$ & 0,863 \\
\hline Não & $68(68,0)$ & $37(54,4)$ & $31(45,6)$ & \\
\hline \multicolumn{5}{|c|}{ Uso de distração durante a refeição (n=131) } \\
\hline Não & $36(27,5)$ & $24(66,7)$ & $12(33,3)$ & 0,037 \\
\hline $\operatorname{Sim}$ & $95(72,5)$ & $44(46,3)$ & $51(53,7)$ & \\
\hline \multicolumn{5}{|c|}{ Autonomia da criança para se servir $(n=126)$} \\
\hline $\operatorname{Sim}$ & $36(28,6)$ & $24(66,7)$ & $12(33,3)$ & 0,042 \\
\hline Não & $90(71,4)$ & $42(46,7)$ & $48(53,3)$ & \\
\hline \multicolumn{5}{|c|}{ Número de alimentos aceitos $(n=113)$} \\
\hline$<15$ alimentos & $35(31,0)$ & $22(62,9)$ & $13(37,1)$ & 0,205 \\
\hline$\geq 15$ alimentos & $78(69,0)$ & $39(50,0)$ & $39(50,0)$ & \\
\hline
\end{tabular}


Os alimentos que mais contribuíram para o consumo de fibras estão descritos na tabela 2. Os 5 principais contribuintes para fibras totais foram: pães, arroz e cereais (20,3\%); frutas (18,9\%); feijões e outras leguminosas (14,1\%); hortaliças $(7,2 \%)$; sucos de frutas $(6,5 \%)$. Para o rank de fibras solúveis e insolúveis, esses mesmos alimentos se repetem, apenas alterando a ordem de contribuição com exceção de salgadinhos e batata frita que aparecem como $4^{\circ}$ contribuinte de fibras solúveis.

Tabela 2 - Alimentos contribuintes para o consumo de fibras totais, fibras solúveis e fibras insolúveis. São Paulo, 2019.

\begin{tabular}{|c|c|c|c|c|c|c|c|c|}
\hline \multicolumn{3}{|c|}{ Fibras totais } & \multicolumn{3}{|c|}{ Fibras solúveis } & \multicolumn{3}{|c|}{ Fibras insolúveis } \\
\hline Rank & Alimentos & $\begin{array}{l}\% \text { de } \\
\text { contribuição }\end{array}$ & Rank & Alimentos & $\begin{array}{l}\% \text { de } \\
\text { contribuição }\end{array}$ & Rank & Alimentos & $\begin{array}{l}\% \text { de } \\
\text { contribuição }\end{array}$ \\
\hline 1 & $\begin{array}{l}\text { Pães, arroz } \\
\text { e cereais }\end{array}$ & 20,3 & 1 & Frutas & 21,5 & 1 & $\begin{array}{l}\text { Pães, arroz } \\
\text { e cereais }\end{array}$ & 22,5 \\
\hline 2 & Frutas & 18,9 & 2 & $\begin{array}{l}\text { Pães, arroz } \\
\text { e cereais }\end{array}$ & 16,8 & 2 & Frutas & 18,3 \\
\hline 3 & $\begin{array}{l}\text { Feijões e out } \\
\text { leguminosas }\end{array}$ & 14,1 & 3 & Sucos de frutas & 11,4 & 3 & $\begin{array}{l}\text { Feijões e out } \\
\text { leguminosas }\end{array}$ & 18,3 \\
\hline 4 & Hortaliças & 7,2 & 4 & $\begin{array}{l}\text { Salgadinhos e } \\
\text { batata frita }\end{array}$ & 8,4 & 4 & Hortaliças & 7,2 \\
\hline 5 & Sucos de fru & 6,5 & 5 & Hortaliças & 7,5 & 5 & Sucos de fru & 5,0 \\
\hline Total & & 67,0 & & & 65,6 & & & 71,3 \\
\hline
\end{tabular}

Em relação aos fatores associados ao consumo de fibras dentro da recomendação (tabela 3), houve associação significativa e positiva para escolaridade dos pais (OR: 5,30; IC 95\% 1,08-25,99; p=0,039). Enquanto uso de distração durante a refeição e falta de autonomia da criança para se servir apresentaram associação significativa negativa (OR: 0,43; IC 95\% 0,19-0,96; p=0,040 e OR: 0,44; IC 95\% 0,20-0,98; p=0,045, respectivamente).

Tabela 3 - Fatores associados ao consumo de fibras dentro da recomendação da SBP. São Paulo, 2019.

\begin{tabular}{llll}
\hline Variáveis & OR (IC 95\%) & Valor de p \\
\hline Sexo & ref & \\
& Feminino & & \\
\multicolumn{1}{c}{ Masculino } & $0,52(0,26-1,06)$ & 0,075 \\
\hline Escolaridade dos pais & ref & \\
Ensino médio & $5,30(1,08-25,99)$ & $\mathbf{0 , 0 3 9}$ \\
\hline Ensino superior & & \\
\hline Aleitamento materno exclusivo & ref & 0,584 \\
Não realizou & $0,79(0,33-1,84)$ & 0,906 \\
\hline 6 meses & $0,95(0,64-2,49)$ & \\
\hline
\end{tabular}




\begin{tabular}{lll}
$\begin{array}{ll}\text { Diagnóstico de dificuldade alimentar } \\
\text { Apetite limitado }\end{array}$ & ref & \\
Fobia alimentar & $0,47(0,07-3,17)$ & 0,435 \\
Seletividade alimentar & $0,74(0,34-1,64)$ & 0,465 \\
Outros & $1,40(0,30-6,56)$ & 0,670 \\
\hline Doença orgânica associada & & \\
Não & ref & 0,807 \\
Sim & $0,92(0,47-1,79)$ & \\
\hline Constipação & & 0,863 \\
Não & ref & \\
Sim & $0,93(0,40-2,16)$ & \\
\hline Uso de distração durante a refeição & & $\mathbf{0 , 0 4 0}$ \\
Não & ref & \\
Sim & $0,43(0,19-0,96)$ & \\
\hline Autonomia da criança para se servir & & $\mathbf{0 , 0 4 5}$ \\
Sim & ref & 0,207 \\
Não & $0,44(0,20-0,98)$ & \\
\hline Número de alimentos aceitos & ref & \\
$<15$ alimentos & $0,59(0,26-1,34)$ & \\
$\geq 15$ alimentos & & \\
\hline
\end{tabular}

\section{DISCUSSÃO}

O presente estudo mostrou que a maioria das crianças (53,8\%) apresentou consumo abaixo da recomendação da SBP, havendo diferenças estatisticamente significativas para escolaridade dos pais, uso de distração durante a refeição e autonomia da criança para se servir. Além disso, os maiores contribuintes para o consumo de fibras totais foram pães, arroz e cereais, seguido de frutas, feijões e outras leguminosas, hortaliças e sucos de frutas. Os contribuintes de fibras solúveis e insolúveis são semelhantes ao rank de fibras totais, com exceção de salgadinhos e batata frita que aparecem como $4^{\circ}$ contribuinte de fibras solúveis. Sobre os fatores associados ao consumo de fibras dentro da recomendação, escolaridade dos pais apresentou associação positiva, enquanto uso de distração durante a refeição e falta de autonomia da criança para se servir apresentaram associação negativa.

Diversos estudos têm demonstrado que a população infantil apresenta baixo consumo de fibras ${ }^{5,12-15}$. Spinelli e colaboradores ${ }^{12}$, ao verificarem o consumo alimentar de crianças entre 6 e 18 meses frequentadoras de creches, encontraram um consumo de fibras que não atendeu às recomendações diárias. A ingestão de fibras nessa amostra atendeu cerca de dois terços das necessidades, podendo representar um risco para constipação intestinal. 0 baixo consumo também foi encontrado por Finn e colaboradores ${ }^{13}$ em estudo com o objetivo de comparar a ingestão de nutrientes e as fontes de fibras entre crianças de até 47,9 meses com baixa e alta ingestão desse nutriente. Além 
disso, verificaram que crianças com ingestão mais alta de fibra alimentar consumiram pelo menos $75 \%$ dos grãos como grãos integrais e consumiram maiores quantidades de frutas e legumes. Dados do estudo ALSPAC 5 - coorte de base populacional - evidenciaram que $77,8-86,2 \%$ das crianças apresentam consumo insuficiente de fibras, valores acima do encontrado no presente estudo (53,8\%). Ainda, mostrou-se que crianças com seletividade apresentam menor ingestão de fibras na dieta quando comparadas às crianças não seletivas (diferença média de -1.4 (IC 95\% -1.6,-1.2) g/dia, p<0.001), além de apresentarem porcentagem mais baixa de fibras provenientes de hortaliças.

Em relação aos alimentos contribuintes para o consumo de fibras, de modo geral, são alimentos de boa qualidade nutricional e fontes de outros nutrientes benéficos, além das fibras. Os contribuintes para fibras totais foram pães, arroz e cereais, frutas, feijões e outras leguminosas, hortaliças e sucos de frutas. Bosscher e colaboradores ${ }^{16}$ apresentaram achados semelhantes, uma vez que pães contribuíram para mais de um terço da ingestão total de fibras alimentares em crianças belgas. As contribuições dos outros grupos alimentares foram 15,1\% para frutas, 14,5\% para alimentos ricos em amido e 13,9\% para vegetais. Em estudo anterior do nosso grupo que mapeou e caracterizou os tipos de alimentos aceitos por crianças com DA, a partir do registro dos alimentos no IA, mostrou-se que essas crianças podem selecionar os alimentos de acordo com o sabor, a cor, consistência, textura e grupo alimentar, muito baseada nos alimentos de maior preferência, sendo que um dos alimentos em que baseiam sua alimentação são produtos de panificação ${ }^{17}$. Relativamente aos salgadinhos e batata frita que aparecem como $4^{0}$ contribuinte de fibras solúveis, estes, são alimentos que possuem baixo teor de fibras, no entanto, sabe-se que crianças geralmente consomem grande parte de suas fibras alimentares a partir de alimentos que apresentam baixa densidade de fibras, mas consomem grandes quantidades desses alimentos ${ }^{4}$. Galloway e colaboradores ${ }^{18}$ estudaram 192 meninas aos 7 anos de idade e compararam três grupos: crianças com neofobia, seletivas e um grupo-controle. Verificaram que tanto as crianças com neofobia $(p<0,05)$, quanto as seletivas $(p<0,01)$ consumiam menos vegetais, excluindo-se a batata frita.

As fibras solúveis são facilmente fermentadas e são solúveis em água, assim, possuem a capacidade de formação de gel, aumentando o volume fecal, provocando a normalização da forma das fezes e facilitando sua eliminação. Já as fibras insolúveis, possuem uma fermentação limitada e não são solúveis em água, assim, elas se movimentam no trato gastrointestinal, promovendo a absorção de fluidos e subprodutos da digestão, causando o aceleramento do movimento intestinal, prevenindo o bloqueio do trato gastrointestinal e a diminuição das evacuações ${ }^{19}$. De modo geral, os alimentos que contêm fibras possuem um terço de fibra solúvel e dois terços de fibra insolúvel20. Já os prebióticos podem ser considerados como todo ingrediente alimentar não digerível que favorece o organismo por estimular o crescimento e/ou atividade da microbiota do cólon, predominando colônias benéficas ${ }^{21}$. Existem indícios de que a colonização do intestino do bebê se inicia no útero, e os micróbios recebidos no nascimento estimularão a colonização que irá evoluir até a idade de 3-6 anos, quando o ecossistema torna-se relativamente estável ${ }^{19,22,23}$. A microbiota intestinal possui papéis importantes na saúde gastrointestinal, como proteção contra patógenos, envolvimento no metabolismo de nutrientes, vitaminas e 
biodisponibilidade de minerais. Além disso, há evidências cada vez maiores de sua participação na proteção contra alguns distúrbios, como por exemplo, doença inflamatória intestinal, diabetes, obesidade e enterocolite necrosante ${ }^{24}$. Nesse cenário, a dieta possui um grande impacto na microbiota intestinal, sendo que a fibra alimentar e os prebióticos são as principais fontes de energia para a colonização desses microrganismos ${ }^{22}$.

Ademais, evidências de estudos em animais e humanos mostram que a microbiota intestinal pode desempenhar um papel importante no comportamento do cérebro e no desenvolvimento cognitivo, produzindo hormônios, fatores imunológicos e metabólitos. microbiota intestinal e o cérebro interagem um com o outro, por isso, o eixo cérebro-intestino tornou-se foco de diversas investigações ${ }^{25}$.

Dessa forma, a ingestão adequada de fibras na infância tem elevada importância para a saúde da criança e para a saúde futura, na vida adulta. Este consumo, logo durante a infância, promove uma adequada saúde gastrointestinal, especialmente como função laxativa; promove a prevenção no ganho de peso; propicia a manutenção dos valores normais de pressão arterial, de glicemia e do perfil lipídico. Reduzindo assim, os riscos de comorbidades na vida adulta ${ }^{26,27 .}$

Sobre os fatores associados ao consumo de fibras dentro da recomendação da SBP, escolaridade dos pais apresentou associação positiva, enquanto uso de distração durante a refeição e a falta de autonomia da criança para se servir apresentaram associação negativa. Sobre esses dois últimos fatores, sabe-se a família tem influência sobre 0 autocontrole da ingestão alimentar na formação de um padrão de comportamento alimentar adequado ou não ${ }^{6}$. Nesse sentido, Satter ${ }^{28}$ propôs um modelo de divisão de responsabilidade o qual os adultos devem se responsabilizar e se comprometer com as decisões sobre o que, quando e onde as refeições são oferecidas; e as crianças devem decidir se devem comer ou não, bem como a quantidade de ingestão em cada refeição. Portanto, a associação negativa para falta de autonomia da criança para se servir indica que a decisão da quantidade de ingestão por parte da criança é uma prática benéfica e que tem sido recomendada na prática clínica no atendimento às $D A$, pois refere-se a autonomia que a criança estabelece na sua alimentação. Por outro lado, o uso de distrações é desencorajado, pois é considerado uma prática coercitiva de alimentação, assim como pressão para comer, práticas de recompensas e punições ${ }^{29}$. Logo, não utilizar distrações durante as refeições faz parte da criação de condições para que a criança desenvolva interesse em se alimentar ${ }^{30}$.

No tocante à escolaridade dos pais, Silva e colaboradores ${ }^{30}$ apontam em artigo de revisão que quanto maior a escolaridade, maior a percepção acerca do desenvolvimento infantil e menos conflituosas as relações com os filhos, o que gera práticas menos punitivas, coercitivas e negligenciais, o que pode influenciar a alimentação, e por consequência, o consumo de macro e micronutrientes, como fibras. Esse resultado também corrobora com 0 achado de Finn e colaboradores ${ }^{13}$, no qual cuidadores de crianças no quartil de baixo consumo de fibras eram menos instruídos e tinham menor renda quando comparados aos do quartil de maior consumo de fibras, especialmente entre crianças de 12 a 23,9 meses. 
O estudo apresenta limitações, sendo elas: (i) o tamanho da amostra que limita a quantidade de variáveis que podem ser usadas nos modelos de regressão; (ii) o consumo de fibras não foi ajustado pela variabilidade intrapessoal; (iii) não foi possível coletar informações mais detalhadas sobre o preparo de alimentos, como, por exemplo, se os sucos foram coados ou não. Apesar disso, é um estudo conduzido em um centro de referência no atendimento às DA que conta com equipe multidisciplinar e protocolos de atendimento e de pesquisa. Também possui como potencialidade a distinção dos tipos de fibras alimentares, uma vez que exercem funções diferentes no organismo.

\section{CONSIDERAÇÕES FINAIS}

A maioria das crianças com dificuldades alimentares apresentou consumo de fibras abaixo da recomendação da Sociedade Brasileira de Pediatria. Os maiores contribuintes para o consumo de fibras totais foram pães, arroz e cereais, seguido de frutas, feijões e outras leguminosas, hortaliças e sucos de frutas. Os contribuintes de fibras solúveis e insolúveis são semelhantes ao rank de fibras totais, com exceção de salgadinhos e batata frita que aparecem como $4^{\circ}$ contribuinte de fibras solúveis. Escolaridade dos pais apresentou associação positiva com o consumo de fibras dentro da recomendação, enquanto uso de distração durante a refeição e falta de autonomia da criança para se servir apresentaram associação negativa.

Apesar de a maioria dos alimentos contribuintes ser considerada de boa qualidade e adequada para uma alimentação infantil variada e saudável (com exceção dos salgadinhos e batata frita), ainda é necessário criar estratégias para aumentar o consumo de fibras por essa população. Conforme observado nas variáveis associadas ao consumo de fibras dentro da recomendação, a dinâmica das refeições e o papel dos pais na alimentação são importantes e devem ser avaliados e orientados durante o acompanhamento das dificuldades alimentares.

\section{AGRADECIMENTOS}

Agradecemos ao Instituto PENSI pelo apoio ao Centro de Dificuldades Alimentares.

\section{REFERÊNCIAS}

1. Sociedade Brasileira de Pediatria - Departamento de Nutrologia. Manual de Alimentação: orientações para alimentação do lactente ao adolescente, na escola, na gestante, na prevenção de doenças e segurança alimentar/ Sociedade Brasileira de Pediatria. Departamento Científico de Nutrologia. 4ª ed. São Paulo; 2018. 
2. Edwards CA, Xie C, Garcia AL. Dietary fibre and health in children and adolescents. Proc Nutr Soc. 2015; 74(3): 292-302.

3. Catalani LA, Kang EMS, Dias MCG, Maculevicius J. Fibras alimentares. Rev Bras Nutr Clín. 2003;18(4): 178-182.

4. Kranz S, Brauchla M, Slavin JL, Miller KB. What do we know about dietary fiber intake in children and health? The effects of fiber intake on constipation, obesity, and diabetes in children. Adv Nutr. 2012; 3(1): 47-53.

5. Taylor CM, Northstone K, Wernimont SM, Emmett PM. Picky eating in preschool children: Associations with dietary fibre intakes and stool hardness. Appetite. 2016; 1; 100: 263-71.

6. Maranhão HS, de Aguiar RC, de Lira DTJ, Sales MUF, Nóbrega NAN. Dificuldades alimentares em pré-escolares, práticas alimentares pregressas e estado nutricional. Rev Paul Pediatr. 2018; 36(1): 45-51.

7. Nogueira-de-Almeida CA, de Mello ED, Filho DR, Maximino P, Fisberg M. Consenso da Associação Brasileira de Nutrologia sobre o uso de suplementos alimentares para crianças com dificuldades alimentares. International Journal Of Nutrology, Int J Nutrol. 2018;11(suppl S1): S4-S15.

8. American Society of Psichiatry. Diagnostic and Statistical Manual of Mental Disorders - DSM V. 2013.

9. Kerzner B. Clinical Investigation of Feeding Difficulties in Young Children: A Practical Approach. Clin Peditr. 2009; 48: 960-5.

10. Maximino P, Machado RHV, Junqueira P, Ciari M, Tosatti AM, Ramos CC, et al. Como acompanhar a criança com dificuldade alimentar em escopo multidisciplinar? Protocolo de atendimento multiprofissional na infância e adolescência - estudo piloto. J Hum Growth Dev. 2018; 26(3): 331-340.

11. Ribeiro LW, Ricci R, Maximino P, Machado RHV, Bozzini AB, Ramos CC, et al. Clinical use of a food inventory to identify maternal underreport on children's food intake: experience of a reference center in Brazil. Nutr Clín Diet Hosp. 2018; 38(1): 81-89. 
12. Spinelli MGN, Goulart RMM, Santos ALP, Gumiero LDC, Farhud CC, de Freitas EB, et al. Consumo alimentar de crianças de 6 a 18 meses em creches. Rev Nutr. 2003; 16(4): 409-414.

13. Finn K, Jacquier E, Kineman B, Storm H, Carvalho R. Nutrient intakes and sources of fiber among children with low and high dietary fiber intake: the 2016 feeding infants and toddlers study (FITS), a cross-sectional survey. BMC Pediatrics. 2019; 19(1): 446.

14. Moshfegh A, Goldman J, Cleveland L. What we eat in America, NHANES 2001-2002. Usual nutrient intakes from food compared to Dietary Reference Intakes. Beltsville, MD: USDA, Agricultural Research Service, 2005.

15. Barbosa RMS, Soares EA, Lanzillotti HS. Avaliação da ingestão de nutrientes de crianças de uma creche filantrópica: aplicação do Consumo Dietético de Referência. Rev Bras Saúde Matern Infant. 2007; 7(2): 159-166.

16. Bosscher D, Caillie-Bertrand MV, Deelstra H. Daily dietary fibre intake of children, 2 to 3 years of age, living in Antwerp, Belgium. Nutrition Research. 2002; 22(12): 1401-1411.

17. Ricci R, Nogueira LR, Maximino P, Machado RHV, Ramos CC, Fisberg M. Repertório alimentar de crianças com dificuldades alimentares baseia-se em doces, sobremesas, produtos de panificação e biscoitos. RASBRAN (no prelo).

18. Galloway AT, Lee Y, Birch LL. Predictors and consequences of food neophobia and pickiness in young girls. J Am Diet Assoc. 2003;103(6):692-698.

19. Eswaran S, Muir J, Chey WD. Fiber and Functional Gastrointestinal Disorders. Am J Gastroenterol. 2013;108(5):718-27.

20. Korczak R, Kamil A, Fleige L, Donovan SM, Slavin JL. Dietary fiber and digestive health in children. Nutr Rev. 2017;75(4):241-259.

21. de Souza VBB, de Vasconcelos LPF, Araújo DGS, Lemos JOM, de Medeiros LSM, Nogueira RBSS, et al. Constipação intestinal em crianças e a importância das fibras alimentares: Uma revisão da literatura. Revista Eletrônica Acervo Saúde. 2019;(21): e561. 
22. Slavin J. Fiber and Prebiotics: Mechanisms and Health Benefits. Nutrients. 2013; 5: 1417-1435.

23. Fuller S, Beck E, Salman H, Tapsell L. New Horizons for the Study of Dietary Fiber and Health: A Review. Plant Foods Hum Nutr. 2016;71(1):1-12.

24. Wegh CAM, Schoterman MHC, Vaughan EE, Belzer C, Benninga MA. The effect of fiber and prebiotics on children's gastrointestinal disorders and microbiome. Expert Rev Gastroenterol Hepatol. 2017;11(11):1031-1045.

25. Wang H, Wang Y. Gut Microbiota-brain Axis. Chin Med J (Engl). 2016;129(19):2373-2380.

26. Anderson JW, Baird P, Davis Jr RH, Ferreri S, Knudtson M, Koraym A, et al. Health benefits of dietary fiber. Nutr Rev. 2009;67(4):188-205.

27. Williams CL, Dietary fiber in childhood. The Journal of Pediatrics. 2006;5: S121-S130.

28. Satter E. Eating competence: definition and evidence for the Satter Eating Competence model. J Nutr Educ Behav. 2007; 39(5 Suppl): S142-53.

29. Machado RHV, Tosatti AM, Malzyner G, Maximino P, Ramos CC, Bozzini AB, et al. Maternal feeding practices among children with feeding difficulties-cross-sectional study in a Brazilian Reference Center. Front Pediatr. 2018;5:286.

30. Silva GAP, Costa KAO, Giugliani ERJ. Infant feeding: beyond the nutritional aspects. J Pediatr. 2016; 92(3 Suppl 1): S2-S7. 